International Journal of Pure and Applied Mathematics

Volume 113 No. 3 2017, 441-453

ISSN: 1311-8080 (printed version); ISSN: 1314-3395 (on-line version)

url: http://www.ijpam.eu

doi: 10.12732/ijpam.v113i3.6

\title{
CHARACTERIZATION OF k-DISJOINTNESS PRESERVING NON-LINEAR OPERATORS BETWEEN BANACH LATTICES
}

\author{
William Feldman ${ }^{1 \S}$, Pramod Singh ${ }^{2}$ \\ ${ }^{2}$ Department of Mathematical Sciences \\ University of Arkansas \\ Fayetteville, AR 72701, USA \\ ${ }^{2}$ Hewlett Packard, Bagmane Tech-Park \\ Embassy Prime CV Raman Nagar \\ Bangalore 560093, INDIA
}

\begin{abstract}
A map T, not necessarily linear, between two Banach lattices $E$ and $F$ is said to kdisjointness preserving if $T\left(f_{0}\right) \wedge T\left(f_{1}\right) \wedge . \wedge T\left(f_{k}\right)=0$, whenever $f_{0}, f_{1}, . ., f_{k}$ are $k+1$ mutually disjoint positive elements in $\mathrm{E}$ and $\mathrm{k}$ is the smallest natural number with this property. Certain k-disjointness preserving maps are characterized in terms of cardinality of subsets of $X$, where $C^{\infty}(X)$ is a representation space of $E$. This then facilitates a decomposition of these kdisjoint non-linear operators into a sum of disjointness preserving operators. Further, given $E$ as represented as functions on $X$ and $F$ as functions on $Y$, the disjointness preserving operator $T$ has the property that $T f(y)=F_{y}\left(f(x)\right.$ where $x$ corresponds to $y$ and $F_{y}$ is an increasing real-valued function defined on the reals or extended reals.
\end{abstract}

AMS Subject Classification: 46B42,47H07,54G05.

Key Words: Disjointness preserving, k-disjointness preserving, extremally disconnected, non-linear operators, Carleman operators, orthogonally additive.

Received: $\quad$ November 15, 2016

Revised: $\quad$ January 10, 2016

Published: $\quad$ March 28, 2017

(c) 2017 Academic Publications, Ltd. url: www.acadpubl.eu

$\S_{\text {Correspondence author }}$ 


\section{Introduction}

We will study disjointness preserving properties for certain non-linear operators between Banach lattices. We will assume that the Banach lattices under consideration have quasi-interior points. We recall that an element $e$ in a Banach lattice $E$ is said to be a quasi-interior point if the order ideal $I(e)$ generated by $e$ (i.e., all elements in $f \in E$ such that $|f|$ is less than a multiple of $e$ ) is dense in $E$.

Now, $I(e)$ can be identified with $C(X)$, all continuous real-valued functions on a compact space $X$ where $e$ is identified with the constant function 1 . In turn, $E$ can be represented as an ideal in $C^{\infty}(X)$, the space of all continuous functions from $X$ to $[-\infty, \infty]$, each finite on a dense subset of $X$. We will refer to $X$ as the representation space for $E$ and identify elements in $E$ with functions on $X$ and we will denote the non-negative elements in $E$ by $E^{+}$. We will be often be concerned with Dedekind complete Banach lattices and in this case the representation space $X$ is extremally disconnected. For details see [8].

We recall that an operator $T: E \rightarrow F$ is a Carleman operator if the image of the unit ball B is order bounded in the universal completion of $F$ (see [6]). For the purposes of this note we will be considering operators $T$ from $E^{+}$to $F^{+}$. In this context, we call $T$ a Carleman operator if the image of positive elements of the unit ball, $B^{+}$, of $E$ is order bounded in the universal completion of $F$. The universal completion of $F$ is identified with $C^{\infty}(Y)$ where $Y$ is extremally disconnected. We define $Y^{\prime}=\{y \in Y: g(y)<\infty\}$ where the image of the unit ball under $T$ is contained in $[-g, g]$ (we choose $g \geq 0$ ).

We specify the formal disjointness property.

Definition 1. Let $T$ be a map from $E^{+}$to $F^{+}$for Banach lattices $E$ and $F$. Then $T$ is said to be a $k$-disjointness preserving map if $T\left(f_{0}\right) \wedge T\left(f_{1}\right) \ldots \wedge$ $T\left(f_{k}\right)=0$, whenever $f_{0}, f_{1}, . ., f_{k}$ are $k+1$ mutually disjoint positive elements in $E$ and $k$ is the minimal positive integer with this property.

1-disjointness preserving coincides with disjointness preserving.

We will be concerned with the following class of operators.

Definition 2. A Carleman operator $T$ between $E^{+}$and $F^{+}$will be called a $C$-operator if $T$ is monotone, subadditive, orthogonally additive and subhomogeous. In more detail, for $f$ and $g$ in $E^{+}$, the operator $T$ satisfies:

(i) $T f \leq T g$ for $0 \leq f \leq g$ (monotone),

(ii) $T(f+g) \leq M(T f+T g)$ for a positive number $M$ (subadditive),

(iii) $T(f+g)=T f+T g$ for $f$ and $g$ orthogonal (orthogonally additive), 
(iv) For each $\alpha>0$, there exist positive constant $M(\alpha)$ so that $T(\alpha f) \leq M(\alpha) T(f)$ (subhomogeneous)

The $\mathrm{C}$-operator is similar but somewhat less restrictive than the $S$-operator in [6]. We also note that $T(0)=0$ a consequence of the orthogonal additivity.

We observe that for a Banach lattice $E$ with representation space $X$, given $0<\gamma \leq 1$, the operator $T_{1}$ defined by $T_{1}(f)=f^{\gamma}$ is a C-operator from $E^{+}$to $E^{+}$.

We first note that $f^{\gamma} \leq f+1_{X}$, where $1_{X}$ denotes the constant function 1 on $X$ and thus $f^{\gamma}$ is in $E$. We can observe directly that $T_{1}$ is monotone, orthogonally additive, and subhomogeneous. Since $(f+g)^{\gamma} \leq 2^{\gamma}\left(f^{\gamma}+g^{\gamma}\right)$ we have $T_{1}$ is subadditive. Further, the Carleman condition is satisfied.

For $E=C(X)$ with the supremum norm topologies we note that for for any $\gamma>0, T_{2}(f)=\left(f^{\gamma}\right)$ is a C-operator.

In this setting $f^{\gamma}$ is also in $E$ and it follows as in the previous example, $T_{2}$ is monotone, subhomogeneous, orthogonally additive and subadditive. Now for the Carleman condition, given $f$ in $\left[0,1_{X}\right]$, we have $f^{\gamma}$ is in $\left[0,1_{X}\right]$.

Both these operators are also disjointness preserving (1-disjointness preserving). For $L$ a linear operator from $E$ to $F$, it follows that $L\left(f^{\gamma}\right)$ is also a C-operator and if $\gamma$ is a positive integer, this would then be an example of an orthogonally additive polynomial (e.g., see [7]).

Since the quasi-interior point corresponds to the constant function 1 in the representation of $E$, we will often write 1 or $\frac{1}{n}$ to represent the constant function $e$ and $\frac{1}{n} e$ respectively.

Definition 3. A C-operator $T$ from $E^{+}$to $F^{+}$will be called $p$-continuous if for each $y$ in $Y^{\prime}$, the sequence $T f_{n}(y)$ converges to $T f(y)$ for $f_{n}$ increasing sequence in $E^{+}$converging to $f$ in $E^{+}$.

We recall that all the linear Carleman operators satisfy this property since the functional $y \circ T$ is continuous for each $y \in Y^{\prime}$.

We will verify that given $T$ a p-continuous C-operator that is disjointness preserving then $T$ is similar to the $T_{1}$ and $T_{2}$ discussed above. Specifically, we show in (Corollary 1) that for each point $y \in Y^{\prime}$, there is a point $x \in X$ so that the functional $y \circ T$ is determined by the value of $f$ at $x$. This in turn tells us that $T f(y)=F_{y}(f(x))$ for $F_{y}$ an increasing real-valued function on the the reals or extended reals. Theorem 3 establishes that every k-disjointness preserving p-continuous C-operator satisfying the continuity condition is a sum of disjointness preserving operators as described above and Theorem 4 establishes the converse. These results utilize the characterization of the support sets for these maps as described in Theorem 2. The last section of this note provides an 
example to elucidate the role of continuity in Corollary 1 and hence int other results of the paper. Theorems 3 and 4 are analogous to results known for linear operators (see [2]) and this study relates to the work [1] and [3].

We will use the definition of a support set as in [5] and include the definition here.

Definition 4. Let $T$ map $E^{+}$to $F^{+}$, where $E$ and $F$ have representation spaces $X$ and $Y$ respectively. Let $y \in Y$. A subset $K_{y}^{T}$ of $X$ will be called the support set at $y$ for $T$ and defined by

$$
K_{y}^{T}=X-\underset{\left\{h \in I(e)^{+}:(T h)(y)=0\right\}}{\bigcup}\{x \in X: h(x)>0\} .
$$

If there is no ambiguity, we abbreviate $K_{y}^{T}$ by $K_{y}$. If $T$ is a linear map and $y$ is in the dense subset $Y^{\prime}$ of $Y$ described below, then $K_{y}$ is just the support of the measure representing the continuous linear functional point evaluation at $y$ composed with $T$. In case $T$ is non-linear, $K_{y}$ is not associated with a measure, but still plays a crucial role in the study of non-linear maps between Banach lattices. It is the cardinality of the sets $K_{y}$ which will be used in Theorem 2.

The following is a restatement of Proposition 2 in [5] for the p-continuous Coperator but in this setting requires a somewhat different proof. If not stated specifically, $X$ will always denote the representation space for $E$ and $Y$ will denote the representation space for the Dedekind completion of $F$. If the order bounded set corresponding to the definition of the Carleman operator is $[0, g]$, then $Y^{\prime}$ will denote $\{y \in Y: g(y)<\infty\}$ which is a dense subset of $Y$ in our subsequent discussion.

Proposition 1. Let $T: E^{+} \rightarrow F^{+}$be a p-continuous C-operator. Then there exists a dense subset $Y^{\prime} \subset Y$ so that for each $y \in Y^{\prime}$ and $f \geq 0$ in $E$, we have

$$
T f(y)=0 \text { if and only if } f \text { restricted to } K_{y} \text { is zero }
$$

or $K_{y}=\emptyset$ in which case $T f(y)=0$ for every $f \in E^{+}$.

Proof. Let $Y^{\prime}$ be defined as above. Assume $K_{y} \neq \emptyset$ and $f \in I(e)$ restricted to $K_{y}$ is not identically zero. Then $\{x \in X: f(x)>0\}$ is not a subset of $K_{y}^{c}=\cup_{T f(y)=0}\{x: f(x)>0\}$ with $T f(y)=0$, i.e., $T f(y)>0$. For $f$ not in $I(e)$, then $T(f \wedge n e)(y)$ for sufficiently large $n$ will be greater than zero and by continuity $T f(y)>0$.

Assume $f \in I(e)$ restricted to $K_{y}$ is zero and $K_{y} \neq \emptyset$. Then we 
have $\overline{\left\{x:\left(\left(f-\frac{1}{n}\right) \vee 0\right)>0\right\}} \subset\{x: f(x)>0\} \subset K_{y}^{c}$. Now by compactness, we have $\overline{\left\{x:\left(\left(f-\frac{1}{n}\right) \vee 0\right)>0\right\}} \subset \cup_{i=1}^{n}\left\{x: f_{i}(x)>0\right\}$ with each $T\left(f_{i}\right)(y)=0$. It follows that $\left(\left(f-\frac{1}{n}\right) \vee 0\right) \leq \alpha \sum_{i=1}^{n} f_{i}(x)$ for some $\alpha>0$. Since $T$ is both monotone and subadditive, we have $T\left(\left(f-\frac{1}{n}\right) \vee 0\right)(y) \leq M \sum_{i=1}^{n} T\left(f_{i}\right)(y)$ for some constant $M$. Thus $T\left(\left(f-\frac{1}{n}\right) \vee 0\right)(y)=0$. By continuity, we have $T f(y)=0$. Again as above, for any $f \in E$ which is zero restricted to $K_{y}$, continuity implies $T f(y)=0$.

If $K_{y}=\emptyset$, then $\bigcup_{\left\{h \in I(e)^{+}:(T h)(y)=0\right\}}\{x \in X: h(x)>0\}=X$ and by compactness, $X$ is equal to a finite union of sets, $\bigcup_{i=1}^{n}\left\{x \in X: h^{i}(x)>\right.$ $0\}$. Now the function 1 is less than $\lambda \sum h_{i}$ for some $\lambda>0$. It follows from the subadditivity and subhomogeneous conditions that $T(1) \leq \hat{\lambda} \sum T\left(h_{i}\right)$ for some $\hat{\lambda}>0$ and thus $T(1)(y)=0$. Now $T f(y)=0$ for any $f \in I(e)^{+}$again by subhomogeneous and since $T(f \wedge n)$ converges to $T f$ by p-continuity, we conclude $T f(y)=0$ for every $f \in E^{+}$.

Theorem 1. Let $E$ be Dedekind complete and $T$ be a p-continuous Coperator from $E^{+}$to $F^{+}$and assume that for $y \in Y^{\prime}$, the set $K_{y}$ is a singleton. Then there is a increasing function $F_{y}$ from $\mathbf{R}$ or $R \cup \infty$ to $\mathbf{R}$ with $F_{y}(0)=0$ so that

$$
T f(y)=F_{y}\left(f\left(K_{y}\right)\right)
$$

for each $f$ in $E^{+}$.

Proof. We will let $z=K_{y}$ and assume that $f(z)=g(z)$ for $f$ and $g$ in $I(e)^{+}=I(e) \cap E^{+}$. Now for $n \in \mathbf{N}$, we have $\left(\left(f-\frac{1}{n}\right) \vee 0\right)(x) \leq g(x)$ for all $x$ in a clopen neighborhood $H$ of $z$. For any $h$ in $I(e)^{+}$, we observe that $T h(y)=$ $T h \chi_{H}(y)+T h \chi_{H^{c}}$ by orthogonal additivity and $T h_{\chi_{H^{c}}}=0$ as a consequence of the previous proposition. Thus $\left.T\left(\left(f-\frac{1}{n}\right) \vee 0\right)(y)=T\left(\left(f-\frac{1}{n}\right) \vee 0\right) \chi_{H}\right)(y)$ and $T(g)(y)=T\left(g \chi_{H}\right)(y)$. Now $\left.\left(f-\frac{1}{n}\right) \vee 0\right) \chi_{H}<g \chi_{H}$ and since $T$ is monotone, we have $T\left(\left(f-\frac{1}{n}\right) \vee 0\right) \leq T\left(g \chi_{H}\right)$ and in turn it follows that $T\left(\left(f-\frac{1}{n}\right) \vee 0\right)(y) \leq$ $T(g)(y)$. Since $T$ is p-continuous, we conclude that $T f(y) \leq T g(y)$. Reversing the roles of $f$ and $g$ establishes $T f(y)=T g(y)$. If $f$ or $g$ in $E^{+}$are not in $I(e)^{+}$, we have $(f \wedge n e)(z)=(g \wedge n e)(z)$ and thus $T(f \wedge n e)(y)=T(g \wedge n e)(y)$. Now p-continuity again implies that $T f(y)=T g(y)$.

Since $T f(y)$ is determined by the value of $f(z)$, we define $F_{y}(r)=T \bar{r}(y)$ where $\bar{r}$ denotes the constant function with value $r$ or if there exists an $f$ with $f(z)=\infty$ then we define $F_{y}(\infty)=T f(y)$. It then follows that $T f(y)=$ $F_{y}(f(z))$ and $F_{y}$ is increasing since $T$ is monotone and $F_{y}(0)=0$ since $T(0)=$ 0 . 


\section{Support Set Characterization of $k$-Disjointness}

We first provide a type of continuity for the map taking each $y$ in $Y^{\prime} \subset Y$ to the subset $K_{y}$ in $X$.

Lemma 1. Let $T$ be a p-continuous C-operator from $E^{+}$to $F^{+}$. If a net $\left\{y_{\alpha}\right\}$ converges to $y$ in $Y^{\prime}$ and $N$ is any open and closed (clopen) set with of $N \cap K_{y} \neq \emptyset$, there exists a $\beta$ so that for $\alpha>\beta, K_{y_{\alpha}} \cap N \neq \emptyset$.

Proof. For $\chi_{N}$, the characteristic function of $N$, we have $T \chi_{N}$ is a Coperator and $\left(T \chi_{N}\right)\left(y_{\alpha}\right) \rightarrow\left(T \chi_{N}\right)(y)$. Now, $T \chi_{N}(y)>0$ by Proposition 1 since $\chi_{N}$ restricted to $K_{y}$ is nonzero. Thus, there exists an index $\beta$ so that if $\alpha>\beta$, then $\left(T \chi_{N}\right)\left(y_{\alpha}\right)>0$ and again by Proposition 1, we have $K_{y_{\alpha}} \cap N \neq \emptyset$.

We now establish a characterization of k-disjointness preserving which will also be used in the proof of Theorem 2 .

Theorem 2. Let $T$ from $E^{+}$to $F^{+}$be a p-continuous C-operator with $E$ Dedekind complete.

If for every $y$ in a dense subset $Y^{\prime}$, the cardinality of $K_{y}$ is less than or equal to $k$, then $T$ is n-disjointness preserving for some $n \leq k$. If in addition, there exists a point $y \in Y^{\prime}$ with the cardinality of $K_{y}$ equal to $k$, then $T$ is $\mathrm{k}$-disjointness preserving.

If $T$ is k-disjointness preserving, then for every $y$ in $Y^{\prime}$, the cardinality of $K_{y}$ is less than or equal to $k$ and there exists a $y \in Y^{\prime}$ with the cardinality of $K_{y}$ equal to k. Further, $\left\{y \in Y^{\prime}:\right.$ cardinality of $\left.K_{y}=k\right\}$ is an open subset of $Y^{\prime}$.

Proof. Assume first that the cardinality of $K_{y}$ is less than or equal to $k$. Given $\left\{f_{i}\right\}_{i=0}^{k}$ of pairwise orthogonal elements in $E$ and $y \in Y^{\prime}$, it follows that one of these functions, call it $f_{j}$, must have $f_{j}$ restricted to $K_{y}$ equal to zero. In turn by Proposition 1 , we know $T f_{j}(y)=0$ implying $\wedge_{i=0}^{k} T f_{i}(y)=0$. Since this is valid for all $y$ in a dense subset $Y^{\prime}$, we conclude that $\wedge_{i=0}^{k} T f_{i}=0$ and therefore $T$ is n-disjointness preserving with $n \leq k$. If in addition, there exists a point $y^{\prime} \in Y^{\prime}$ with the cardinality of $K_{y^{\prime}}$ equal to $k$, then there exist $g_{0}, \ldots, g_{k-1}$ pairwise disjoint with each $g_{i}$ nonzero at some point of $K_{y^{\prime}}$. Thus again by Proposition $1, T g_{i}\left(y^{\prime}\right) \neq 0$ for each $i$ and thus $\wedge_{i=0}^{k-1} T g_{i} \neq 0$ establishing that $k$ is minimal, i.e., $T$ is $\mathrm{k}$-disjointness preserving.

Let $T$ be k-disjointness preserving. Assume to the contrary that there is a point $y \in Y^{\prime}$ with the cardinality of $K_{y}$ greater than $k$. Then, as above, there exists $g_{0}, \ldots, g_{k}$ pairwise disjoint with each $g_{i}$ nonzero at some point of $K_{y}$ and in 
turn, $\wedge_{i=0}^{k} T g_{i} \neq 0$ contradicting k-disjointness preserving. Thus the cardinality of each $K_{y}$ for $y \in Y^{\prime}$ is less than or equal to $k$. If it were the case that for every $y \in Y^{\prime}$ the set $K_{y}$ has strictly fewer than $k$ points, then as above any $\left\{f_{i}\right\}_{i=0}^{k-1}$, a collection of pairwise disjoint functions, would have at least one function $f_{j}$ that must vanish on $K_{y}$ implying that $T f_{j}(y)=0$ and $\wedge_{i=0}^{k-1} T f_{i}=0$. Thus $k$ would not be minimal. We conclude that there exists at least one point $y$ in $Y^{\prime}$ with the cardinality of $K_{y}$ equal $k$.

Let $V=\left\{y \in Y^{\prime}:\right.$ cardinality of $K_{y}$ is $\left.k\right\}$. To show the complement of $Y^{\prime}-V$ is closed in $Y^{\prime}$, assume there exists a net $\left\{y_{\alpha}\right\} \subset Y^{\prime}-V$ convergent to $y$ in $V$. Let $N_{0}, N_{1}, \ldots, N_{k-1}$ be disjoint open and closed (clopen) sets containing each of the points in $K_{y}$. Now since $K_{y} \cap N_{0} \neq \emptyset$, Lemma 1 implies that there exists an $\alpha_{0}$ so that for all $\alpha>\alpha_{0}$, the set $K_{y_{\alpha}} \cap N_{0}$ is not empty. In turn, there exists an $\alpha_{1}>\alpha_{0}$ with $K_{y_{\alpha}} \cap N_{1} \neq \emptyset$ for $\alpha>\alpha_{1}$. Continuing, we find $\alpha_{i}>\alpha_{i-1}$ with $K_{y_{\alpha}} \cap N_{i} \neq \emptyset$ for each $\alpha>\alpha_{i}$ and $2<i \leq(k-1)$. We note that $y_{\alpha}$ is in $Y^{\prime}-V$, and thus the cardinality of each $K_{y_{\alpha}}$ is less than or equal to $(k-1)$. Therefore, for $\alpha>\alpha_{k-1}$, the set $K_{y_{\alpha}} \cap N_{i} \neq \emptyset$ for $0 \leq i \leq k-1$ is impossible.

We now know that the support set $K_{y}$ for a disjointness preserving operator is one point or the empty set for all $y$ in $Y^{\prime}$. Thus in light of the previous two theorems, we are able to establish the following:

Corollary 1. Let $T$ from $E^{+}$to $F^{+}$be a disjointness preserving pcontinuous C-operator with $E$ Dedekind complete and $Y^{*}=\left\{y \in Y^{\prime}: T f(y) \neq\right.$ 0 for some $\left.f \in E^{+}\right\}$. There exists a continuous map $\mu$ from $Y^{*}$ to $X$ and an increasing real-valued function $F_{y}$ on $[0, \infty)$ or $[0, \infty]$ with $F_{y}(0)=0$ so that

$$
T f(y)=F_{y}(f(\mu(y)))
$$

for $y \in Y^{*}$.

Proof. We need only verify that $\mu$ is continuous but this is a consequence of Lemma 1. Indeed if a net $\left(y_{\alpha}\right)$ is convergent to $y$ in $Y^{*}$ and $N$ is any clopen neighborhood of $y$, then Lemma 1 implies $\mu\left(y_{\alpha}\right)=K_{y_{\alpha}}$ is eventually in $N$.

\section{3. $k$-Disjointness as Sum of 1-Disjointness}

Theorem 3. Let $T$, a map from $E^{+}$to $F^{+}$, be a p-continuous C-operator with $E$ and $F$ Dedekind complete. If $T$ is k-disjointness preserving, then there 
exist disjointness preserving C-operators $T_{1}, T_{2} \ldots, T_{k}$ so that for each $f \geq 0$ in $\mathrm{E}$

$$
T f=\sum_{i=1}^{k} T_{i}(f)
$$

Proof. We proceed by induction. There is nothing to prove if $T$ is disjointness preserving. Let us assume the result is true for i-disjointness preserving for $2 \leq i \leq k-1$. Given $T$ is $\mathrm{k}$-disjointness preserving, then by the previous Theorem we have $V=\left\{y \in Y^{\prime}\right.$ : cardinality of $K_{y}$ is $\left.k\right\}$ is open in $Y^{\prime}$.

For $y \in V$ and $N_{0}, N_{1}, \ldots, N_{k-1}$ pairwise disjoint clopen (open and closed) neighborhoods of the points in $K_{y}$, we claim there exists a clopen neighborhood $W$ of $y$ such that for every $z$ in $W \cap Y^{\prime}$, the set $K_{z} \cap N_{0}$ is a one point set. If false, there exists a net $\left\{y_{\alpha}\right\}$ in $V$ convergent to $y$ with $y_{\alpha} \neq y$ and the cardinality of $K_{y_{\alpha}} \cap N_{0}$ not equal to 1 . By Lemma 1 we can assume that $K_{y_{\alpha}} \cap N_{0} \neq \emptyset$. Thus, there is an $\alpha_{0}$ so that for $\alpha>\alpha_{0}$, the cardinality of $K_{y_{\alpha}} \cap N_{0}$ exceeds 1 . Again by Lemma 1 , there is an $\alpha_{1}>\alpha_{0}$ so that for $\alpha>\alpha_{1}$, we have the cardinality of $K_{y_{\alpha}} \cap N_{1}$ greater than or equal to 1 . Continuing for $2<i \leq k-1$, there is an $\alpha_{i}>\alpha_{i-1}$ so that for $\alpha>\alpha_{i}$, the cardinality of $K_{y_{\alpha}} \cap N_{i}$ greater than or equal to 1 . This is not possible for $\alpha>\alpha_{k-1}$ since each $K_{y_{\alpha}}$ has $k$ points establishing our claim for the clopen set $W$. By repeating this argument, we can find $W$, a clopen neighborhood of $y$ such that $K_{z} \cap N_{i}$ is a one point set for every $z \in W \cap Y^{\prime}$ and $0 \leq i \leq k-1$. We will denote $N_{0}$ by $N$ and verify that $\chi_{W} T \chi_{N}$ is disjointness preserving. Given $f$ and $g$ positive and disjoint in $E$ and say for some $t$ in $Y^{\prime},\left(\chi_{W} T \chi_{N} f\right)(t)>0$. Since $t$ must be in $W, K_{t} \cap N$ is one point. If we denote this points by $x$, then $\chi_{N} f$ is positive at $x$ and so $\chi_{N} g$ at $x$ must be zero and therefore $\left(\chi_{W} T \chi_{N} g\right)(t)=0$ establishing disjointness. We also observe that $\chi_{W} T \chi_{N}$ is an C-operator. It is clearly monotone, subadditive, orthogonally additive, and subhomogeneous since $T$ has this property. Further, $\chi_{W} T \chi_{N}$ satisfies the Carleman condition for the same order bounded set as required by the condition on $T$ and $T$ is clearly also p-continuous.

Let $\mathcal{W}$ denote a collection of pairwise disjoint clopen sets $W$ with $W \cap Y^{\prime}$ contained in $V$ which have a corresponding clopen set $N$ in $X$ with $\chi_{W} T \chi_{N}$ disjointness preserving and $K_{z} \cap N_{i}$ for $0 \leq i \leq k-1$ a one point set for all $z \in W \cap Y^{\prime}$. Now the set $\mathcal{A}$ of all such collections $\mathcal{W}$ is partially ordered by inclusion. Given a chain $\mathcal{C}$ in $\mathcal{A}$, the collection $\{W: W \in \mathcal{W}$ for some $\mathcal{W}$ in $\mathcal{C}\}$ is an upper bound for $\mathcal{C}$ so that by Zorn's Lemma we have a maximal element, call it $\mathcal{W}^{*}$

We claim the $\cup\left\{W \cap Y^{\prime}: W \in \mathcal{W}^{*}\right\}$ is dense in $V$. If not, there is a clopen neighborhood $D$ of a point $y$ in $V$ such that $D \cap Y^{\prime}$ is contained in $V$ and 
disjoint from $\cup\left\{W \cap Y^{\prime}: W \in \mathcal{W}^{*}\right\}$. By our previous constructions, we could find a clopen set $\hat{W} \subset D$ with $y \in \hat{W}$ and corresponding clopen set $\hat{N}\left(\hat{N}=N_{0}\right)$ so that $\chi_{\hat{W}} T \chi_{\hat{N}}$ is disjointness preserving and $K_{z} \cap N_{i}$ for $0 \leq i \leq k-1$ a one point set for all $z \in \hat{W} \cap Y^{\prime}$. Therefore, $\hat{W}$ adjoined to $\mathcal{W}^{*}$ would be strictly greater than $\mathcal{W}^{*}$ contradicting the maximality. Thus the density is established. For each $W$ in $\mathcal{W}^{*}$, we find a corresponding $N$ and consider the operator $L$ defined by

$$
L(f)=\bigvee_{W \in \mathcal{W}^{*}}\left(\chi_{W} T \chi_{N}(f)\right)
$$

for each $f \geq 0$ in $E$.

We claim $L$ is an C-operator. We first note that since $F$ is Dedekind complete, $L(f)$ is in $F$. It is evident that $L$ is monotone and

$$
\begin{gathered}
L(f+g)= \\
\vee\left(\chi_{W} T \chi_{N}(f+g)\right) \leq \vee M\left(\chi_{W} T \chi_{N}(f)\right)+\vee M\left(\chi_{W} T \chi_{N}(g)\right)= \\
M(L(f)+L(g)),
\end{gathered}
$$

where $M$ is the constant corresponding to the subadditivity of $T$. Thus $L$ is subadditive. Given $f$ and $g$ positive and disjoint, we noted that each $\chi_{W} T \chi_{N}$ is orthogonally additive, i.e., $\chi_{W} T \chi_{N}(f+g)=\chi_{W} T \chi_{N}(f)+\chi_{W} T \chi_{N}(g)$. For any $h \in E$ and fixed $\hat{W} \in \mathcal{W}^{*}$, we claim $\chi_{\hat{W}} L(h)=\chi_{\hat{W}} T \chi_{\hat{N}}(h)$. The functions $\chi_{\hat{W}} L(h)$ and $\chi_{\hat{W}} T \chi_{\hat{N}}(h)$ differ only on $\hat{W}$ since $\hat{W}$ is clopen. Assume to the contrary that $\chi_{\hat{W}} L(h)$ is greater than and not equal to $\chi_{\hat{W}} T \chi_{\hat{N}}(h)$. Now $\vee\left\{\chi_{W} T \chi_{N}(h): W \in \mathcal{W}^{*}-\hat{W}\right\}$ is disjoint from $\chi_{\hat{W}} T \chi_{\hat{N}}(h)$. Then for $y \in \hat{W}$, we have $L(h)$ is greater than and not equal to

$$
\vee\left\{\chi_{W} T \chi_{N}(h): W \in \mathcal{W}^{*}-\hat{W}\right\} \vee \chi_{\hat{W}} T \chi_{\hat{N}}(h)
$$

contradicting the construction of $L$ as a supremum. It now follows for $f$ and $g$ disjoint and $y$ a point in $\cup\left\{W \cap Y^{\prime}: W \in \mathcal{W}^{*}\right\}$, say $y \in \hat{W}$, we have

$$
\begin{gathered}
L(f+g)(y)=\left(\bigvee_{W \in \mathcal{W}^{*}} \chi_{W} T \chi_{N}(f+g)\right)(y)=\chi_{\hat{W}} T \chi_{\hat{N}}(f+g)(y) \\
=\chi_{\hat{W}} T \chi_{\hat{N}}(f)(y)+\chi_{\hat{W}} T \chi_{\hat{N}}(g)(y)=L(f)(y)+L(g)(y) .
\end{gathered}
$$

This is valid for all $y$ in the set $\cup\left\{W: W \in \mathcal{W}^{*}\right\}$. Further, for all $h \in E^{+}$, we have $L(h)(y)=0$ for $y \in Y-\bar{V}$. Thus the above equation is valid for all $y$ in a dense subset of $Y$ and hence valid for all $y$ in $Y$ establishing the orthogonal additivity. We know $T(\alpha f) \leq M(\alpha) T(f)$ which implies that 
$\chi_{\hat{W}} T \chi_{\hat{N}}(\alpha f) \leq M(\alpha) \chi_{\hat{W}} T \chi_{\hat{N}}(f)$. Taking suprema, we have $L(\alpha f)<M(\alpha) L(f)$ as desired. We also note that for $y$ in $\cup\left\{W \cap Y^{\prime}: W \in \mathcal{W}^{*}\right\}$ the set $K_{y}$ (i.e., $\left.K_{y}^{L}\right)$ is a singleton since

$L(f)(y)=\chi_{W} T \chi_{N}(f)(y)$ and $K_{y}$ is empty for $y$ in $Y^{\prime}-\bar{V}$. Thus $L$ is disjointness preserving by Theorem 1 . Further, $L$ satisfies the Carleman condition for the same order bounded set that exists for $T$ establishing that $L$ is a C-operator. We verify that $L$ is also p-continuous. Given $f_{n}$ increasing to $f, y \in Y^{\prime}$ and $\epsilon>0$, we have $T\left(\chi_{N} f_{n}\right)(y)>T\left(\chi_{N} f\right)(y)-\epsilon$ for large $n$ since $T$ is p-continuous. In turn $\left.\left(\chi_{W} T \chi_{N}\right) f_{n}\right)(y)>\left(\chi_{W} T \chi_{N}\right) f(y)-\epsilon$. Taking suprema, we conclude that $L f_{n}(y)$ converges to $L f(y)$ establishing p-continuous.

We next establish that $T-L$ is an C-operator. We note that for $\hat{y} \in \cup\left\{W \cap Y^{\prime}: W \in \mathcal{W}^{*}\right\}$, say $\hat{y} \in \hat{W} \cap Y^{\prime}$, we have the disjoint clopen neighborhoods $N_{0}, N_{1} \ldots, N_{k-1}$ of the points in $K_{\hat{y}}$ (i.e., $K_{\hat{y}}^{T}$ ) as mentioned above. For any $f$ in $E^{+}$, the function $\sum_{i=0}^{k-1} \chi_{N_{i}} f$ agrees with $f$ on $K_{\hat{y}}$. Further, for $y$ in $\hat{W}$,

$T f(y)=T\left(f-\sum_{i=0}^{k-1} \chi_{N_{i}} f+\sum_{i=0}^{k-1} \chi_{N_{i}} f\right)(y)=T\left(f-\sum_{i=0}^{k-1} \chi_{N_{i}} f\right)(y)+T\left(\sum_{i=0}^{k-1} \chi_{N_{i}} f\right)(y)$

by orthogonal additivity and $T\left(f-\sum_{i=0}^{k-1} \chi_{N_{i}} f\right)(y)=0$ by Proposition 1 . This gives us $T f(y)=T\left(\sum_{i=0}^{k-1} \chi_{N_{i}} f\right)(y)$. Again orthogonal additivity implies

$$
T f(y)=\sum_{i=0}^{k-1} T \chi_{N_{i}}(f)(y) .
$$

Since we know from above that $L(f)(y)=T \chi_{N_{0}}(f)(y)$, we observe that $(T-L) f(y)=\sum_{i=1}^{k-1} T \chi_{N_{i}}(f)(y)$ for $y \in \hat{W}$ and $(T-L)(f)(y)=T f(y)$ for $y \in Y^{\prime}-\bar{V}$. It follows that $T-L$ is monotone, subadditive, orthogonally additive, and subhomogeneous. Again the same order bounded set will suffice for $T-L$ establishing that $T-L$ is a C-operator. Again, $T-L$ is p-continuous.

We now argue that $T-L$ is $(\mathrm{k}-1)$-disjointness preserving which will complete the induction proof. By Theorem 1, it is sufficient to show that for all $y$ in a dense subset of $Y^{\prime}$, the cardinality of $K_{y}^{T-L}$ is less than or equal to $k-1$ and for one point, the cardinality of $K_{y}^{T-L}$ is equal to $(k-1)$. Now for each $y \in \cup\left\{W \cap Y^{\prime}: W \in \mathcal{W}^{*}\right\}$ our observation above shows that

$$
K_{y}^{T-L}=K_{y}^{T} \cap \cup_{i=1}^{k-1} N_{i}
$$

which has exactly $(k-1)$ points and for $y \in\left(Y^{\prime}-\bar{V}\right)$ each $K_{y}^{T-L}$ has less than or equal to $k-1$ points as desired confirming the $T-L$ is (k-1)-disjointness preserving. 
Lemma 2. Let $T$ and $L$ be C-operators. There is a dense subset $D$ of $Y$, so that for each $y$ in $D$,

$$
K_{y}^{T+L}=K_{y}^{T} \cup K_{y}^{L}
$$

Proof. Let $D$ be the set $Y^{\prime}$ corresponding to the operator $T+L$. Let $\hat{x}$ be an element of the complement of $K_{y}^{T} \cup K_{y}^{L}$ which is equal to $\tilde{K}_{y}^{T} \cap \tilde{K}_{y}^{L}$. Then there exists a positive $f$ in $E^{+}$with $f(\hat{x})>0$ and $T f(y)=0$ and a positive $g$ in $E^{+}$with $g(\hat{x})>0$ and $L g(y)=0$. Then $(f \wedge g)(\hat{x})>0$ and since the operators are monotone, $(T+L)(f \wedge g)(y)=0$ showing $\hat{x}$ is in the complement of $K_{y}^{T+L}$. Now for $\hat{x}$ in the complement of $K_{y}^{T+L}$, we have a positive $f$ in $E$ with $f(\hat{x})>0$ and $(T+L) f(y)=0$. Thus $T f(y)=0$ and $L f(y)=0$ so that $\hat{x}$ is in both $\tilde{K}_{y}^{T}$ and $\tilde{K}_{y}^{L}$ establishing the equality.

Theorem 4. For $k \in N$, the sum of $k$ disjointness preserving operators is an n-disjointness preserving for some $n \leq k$.

Proof. Let $L$ denote the sum of $n$ disjointness preserving operators for $n \leq$ $k$. Applying Lemma 2, we observe that for every $y$ in a dense subset of $Y$ the set $K_{y}^{L}$ has cardinality less than or equal to $\mathrm{k}$ since for any disjointness preserving operator, $K_{y}$ has at most one point by Theorem 1. Again applying Theorem 1, we conclude that $L$ is $\mathrm{k}$-disjointness preserving with $n \leq k$.

\section{Discontinuous Disjointness Preserving Operator}

We will discuss an example of a disjointness preserving $\mathrm{C}$-operator which is not p-continuous and for which the conclusion of Corollary 1 is not valid.

We we construct a map $T$ from $C(\beta N)$ to $[0, \infty)$. Let $z$ be a fixed point in $\beta N-N$

$$
T f=\left\{\begin{array}{cl}
f(z) & \text { if } f(z)<1 \\
\left(\left(\lim \left(\frac{(f(n)-1) \vee 0}{n}\right)+1\right) \wedge 2\right. & \text { if } f(z)=1 \\
f(z)+2 & \text { if } f(z)>1
\end{array}\right\}
$$

We will know verify that $T$ is a $C$-operator. It is clear that $T$ is monotone. To verify subadditivity, assume first that $T(f+g)<1$, i.e., $(f+g)(z)<1$, then it follows that $T(f+g)=T f+T g$. For $(f+g)(z)>1$, we have $T(f+g)=$ $(f+g)(z)+2 \leq T f+T g+2 \leq 4(T f+T g)$ since $T f+T g \geq 1$. To verify orthogonal additivity, let $f \wedge g=0$. If $f(z) \neq 0$, then there is a clopen neighborhood $H$ of $z$ with $f$ strictly positive restricted to $H$ so that $g$ is zero on $H^{c}$ and in turn 
$T(f+g)=T(f)=T(f)+T(g)$. Given $f(z)=0$, if $g(z) \neq 0$, then $f$ on a clopen neighborhood of $z$ is zero so that again $T(f+g)=T g=T f+T g$. If $f(z)=g(z)=0$, then $T(f+g)=0=T f+T g$. To show subhomogeneous, we first consider the case that $\alpha f(z) \neq 1$. If $\alpha f(z)<1$, then $T(\alpha f)=\alpha f(z) \leq$ $\alpha T f$. If $\alpha f(z)>1$, then $T(\alpha f)=\alpha f(\infty)+2 \leq \alpha T(f)+2 \leq(3 \alpha) T(f)$ since $1<\alpha f(z)<\alpha T(f)$. For the case of $\alpha f(z)=1$, we have $T(\alpha f) \leq 2 \leq 2 \alpha T f$ again since $\alpha T f>\alpha f(z)>1$.

We now observe that $T$ is disjointness preserving. Let $f \wedge g=0$. If $T f \neq$ 0 , then $T g=0$ as we noted above in proving orthogonal additivity. Then $T f \wedge T g=0$. Clearly if $T f=0$, we also have $T f \wedge T g=0$. Therefore we have $T$ is a C-operator. Further, the support set $K$ for $T$ is $z$. If $p \neq z$ is in $\beta N$, then there exists a clopen $H$ disjoint from $\{z\}$ containing $p$. Then $T\left(\chi_{H}\right)=0$ so that $H$ is a subset of $K^{c}$ and thus $\{z\}^{c} \subset K^{c}$ or $K \subset\{z\}$. We observe that the argument in Proposition 1 establishing that if $K_{y}=\emptyset$ then $T f(y)=0$ for all $f$ is still valid. Since $T$ is not zero, we have $K=\{z\}$. We can see directly that we have $T f=0$ if and only if $f(K)=0$ (the same conclusion as in Proposition 1). However, clearly $T$ is not p-continuous and $T f$ is not determined by the value of $f$ at $\infty$, that is, the conclusion of Corollary 1 as well as Theorem 1 are not valid for $T$.

\section{References}

[1] Abramovich, Y.A., Multiplicative representation of disjointness preserving operators, Indag. Math. 45 (1983) 265-279, doi: 10.1016/1385-7258(83)90062-8.

[2] Benyamini, Y., Lassalle, S. and Llavona, J., Homogeneous orthogonally additive polynomials on Banach lattices, Bull. London Math. Soc. 38, 3 (2006) 459-469.

[3] Bernau, S.J., Huijsmans, C.B. and De Pagter, B., Sums of Lattice Homomorphisms, Proc. Amer Math. Soc. 115, 1 (1992) 151-156, doi: 10.1090/S0002-9939-1992-1086322-8.

[4] Carothers, D.C. and Feldman, W.A., Sums of homomorphisms on Banach lattices, J. Operator theory 24 (1990) 337-349

[5] Feldman, W.A., Separation properties for Carleman operators on Banach lattices, Positivity 7 (2003) 41-45 doi: 10.1023/A:1025818931354.

[6] Feldman, W.A and Singh, P., Positively Decomposable maps between Banach lattices, Positivity 12 (2008) 495-502, doi: 10.1007/s11117-007-2115-5.

[7] Grobler, J.J. and Van Eldik, P., Carleman operators on Riesz spaces, Indag. Math. 45 (1983) 421-433, doi: 10.1016/0019-3577-90-90031-H.

[8] Pérez-García, David and Ignacio Villanueva, Orthogonally additive polynomials on spaces of continuous functions, J. Math. Anal. Appl. 306, 1 (2005) 97-105, doi: 10.1016/j.jmaa.2004.12.036. 
[9] Schaefer, H.H., Banach lattices and positive operators, Berlin, Heidelberg, New York: Springer-Verlag (1974), doi: 10.1007/978-3-642-65970-6. 
